\title{
Custom Acetabular Cages Offer Stable Fixation and Improved Hip Scores for Revision THA With Severe Bone Defects
}

\author{
Huiwu Li MD, Xinhua Qu MD, Yuanqing Mao MD, Kerong Dai MD, \\ Zhenan Zhu MD
}

Received: 8 July 2015/Accepted: 2 October 2015/Published online: 14 October 2015

(C) The Association of Bone and Joint Surgeons(B 2015

\begin{abstract}
Background Revision THA is particularly challenging in hips with severe acetabular bone loss. When the extent or geometry of the acetabular bone loss precludes morestraightforward techniques such as jumbo hemispheric cementless shells, reconstruction with morselized allograft protected by a custom cage may offer an alternative, but, to our knowledge, few series have reported on results with this approach.

Questions/purposes For patients with severe (Paprosky IIIB) defects, we asked: do individualized custom cages result in (1) improved Harris hip scores; (2) restoration of
\end{abstract}

Each author certifies that he or she, or a member of his or her immediate family, has no funding or commercial associations (eg, consultancies, stock ownership, equity interest, patent/licensing arrangements, etc) that might pose a conflict of interest in connection with the submitted article. One the authors (HL) have received funding from the Shanghai Natural Science Foundation (Grant No. 15ZR1424800) and Scientific Research Foundation for the Returned Overseas Chinese Scholars, Ministry of Education of China (Grant No. 201550002).

All ICMJE Conflict of Interest Forms for authors and Clinical Orthopaedics and Related Research ${ }^{\mathbb{R}}$ editors and board members are on file with the publication and can be viewed on request.

Clinical Orthopaedics and Related Research ${ }^{\mathbb{R}}$ neither advocates nor endorses the use of any treatment, drug, or device. Readers are encouraged to always seek additional information, including FDAapproval status, of any drug or device prior to clinical use. Each author certifies that his or her institution approved the human protocol for this investigation, that all investigations were conducted in conformity with ethical principles of research, and that informed consent for participation in the study was obtained.

H. Li, X. Qu, Y. Mao, K. Dai, Z. Zhu (ه)

Department of Orthopaedics, Shanghai No. 9th People's

Hospital, Shanghai Jiao Tong University School of Medicine,

Shanghai 200011, China

e-mail: zhenan_zhu@126.com hip center; and (3) a low incidence of surgical complications?

Methods Twenty-six patients (26 hips) with a massive acetabular defect were involved in this study from 2003 to 2013. During this period, one patient was lost to followup and one died, leaving 24 patients (eight males, 16 females) in this retrospective analysis. The customized cages were individualized to each patient's bone defect based on rapidprototype three-dimensional printed models. Mean followup was 67 months (range, 24-120 months). Harris hip scores were assessed before surgery and at each followup. Postoperative radiographs were evaluated for cage position, migration, and graft incorporation. Complications and reoperations were assessed by chart review.

Results The mean Harris hip score improved from 36 (SD, 8; range, 20-49) to 82 (SD, 18; range, 60-96) $(\mathrm{p}<$ $0.001)$. Individualized custom cages resulted in generally reliable restoration of the hip center. No rerevisions have been performed. None of the cups showed radiographic migration, but one cage was believed to be loose, based on a circumferential 2-mm radiolucent line. Cancellous allografts appeared to be incorporated in 23 of 24 patients. One deep infection and one superficial infection were observed and treated with irrigation, débridement, and vacuumsealing drainage. One dislocation and one suspected injury of the superior gluteal nerve also were observed and treated conservatively.

Conclusions Individualized custom cages using rapid prototyping and three-dimensional printing appeared to provide stable fixation and improved hip scores at shortterm followup in this small, single-center series. As further improvements in the design and manufacturing process are made, future studies should evaluate larger patient groups for longer times, and, ideally, compare this approach with alternatives for these complex bone defects. 
Level of Evidence Level IV, therapeutic study.

\section{Introduction}

The reconstruction of massive acetabular bone defects remains a challenging problem in revision THA $[4,14,27]$. Multiple treatment options have been proposed, including massive structural allografts, oversized hemispheric cups, impaction bone grafting, and acetabular reconstruction cages. However, none of those options has been shown to predictably yield favorable results in the setting of massive periacetabular bone loss. Oversized hemispheric cups have shown good results [9]; however, the extent or geometry of the acetabular bone loss may preclude this option in the presence of more-severe bone defects [12]. Impaction grafting techniques may be inadequate for stabilizing pelvic discontinuity and do not typically provide adequate acetabular component stability in cases in which major portions of the anterior and posterior columns are absent [30, 34, 35]. Even conventional off-the-shelf cages with structural allografts have had reported relatively high rates of short- and medium-term mechanical failures (from $12.5 \%$ to $37.5 \%$ ) [11, 13, 23, 28, 29, 31, 37]. Furthermore, the variability in the shape of the pelvis and in the size and shape of acetabular defects often results in a relatively poor fit of the off-the-shelf cage to host bone; this is important, because achieving cage stability on host bone is the main challenge in using acetabular reconstruction cages. Revision methods that result in good initial stability on host bone achieve good long-term results, whereas methods that rely on bone graft or compromised host bone for stability have dramatically increased rates of failure $[3,5,6,22,26]$. Cup-cage constructs with a porous metal acetabular component covered by a cage fixed proximally and distally have shown good short and mid-term results, but longerterm followup is necessary $[1,2]$.

A custom cage can provide individualized treatment based on the extent of the acetabular bone defect. Such cages can span a pelvic discontinuity while allowing for direct support of the implant by the host bone to ensure initial stability and maintaining the hip center or returning it to its anatomic location. Production of an individualized custom cage begins with a CT scan of the patient's pelvis. From these data, a life-sized model of the pelvis is created using a rapid-prototyping machine. The model then is used as a basis for construction of an individualized custom cage with three-dimensional (3-D) printing used to create custom augments as needed. Individualized custom cages have been used to treat massive acetabular bone defects since the 1990s [7, 20]. Although some series have reported results with this method $[7,15,32,36]$, these studies generally did not explore the rationale for this approach, describe in detail the techniques of customization and 3-D printing, or provide longer-term results that show advantages of individualized custom cages for complex acetabular revision.

We therefore sought to evaluate a group of patients with severe (Paprosky IIIB) defects to determine whether individualized custom cages result in (1) improved Harris hip scores, (2) restoration of the hip center, and (3) a low incidence of surgical complications.

\section{Patients and Methods}

\section{Patients}

This study was approved by the ethics committee in our institution. For patients presenting with suspected Paprosky IIIB acetabular defects [21], we first made rapid-prototype models according to their CT data before we decided how to do the revision surgery. If the rapid-prototype models indicated the superior dome and the posterior and anterior columns of the acetabulum were likely to provide appropriate support for a jumbo cup or a commercially available cage, we used those options; otherwise, a customized cage, which contacts the host bone directly via the modified crest or augments, was ordered. Based on this evaluation process, between 2003 and 2013, we treated 72 patients for Paprosky IIIB acetabular defects. Of these, 26 (36\%) were treated with acetabular revision using a custom cage. The remainder of the patients were treated with a hemispheric cup with augments or commercially available cages according to the above indications. During this period, one patient was lost to followup and one patient died, leaving a final sample of 24 patients (eight males, 16 females) for analysis in this retrospective study. A pelvic discontinuity was identified in four patients. We also classified the defect using the American Academy of Orthopaedic Surgeons (AAOS) classification as reported by D'Antonio et al. [6]. Of the 26 patients treated with a custom cage, 22 had AAOS type III defects and four had AAOS type IV defects.

Followup took place at a mean of 67 months (range, 24120 months). The mean age of the patients at the time of revision surgery was 65 years (range, 54-79 years). In this study, 21 of the 24 patients were evaluated within the last 5 years, and hip scores and other parameters were recorded at the latest followup. We contacted the other three patients by telephone.

All patients showed marked disability preoperatively and required ambulatory aids. Twenty-three operations were first-time revisions, whereas one was a rerevision. Defects of this severity are not unusual at the time of an initial revision THA in China since many patients come from remote areas and may not present for care until they have very severe pain or dysfunction. By then, the bone 
loss sometimes is quite severe. The diagnoses necessitating the original THA was developmental dysplasia in eight patients, osteoarthritis in seven, femoral neck or acetabular fracture in four, avascular necrosis in four, and rheumatoid arthritis in one. Cemented cups were used at the time of primary THA in 14 patients and uncemented cups were used in 10 patients. The reason for revision was aseptic loosening in all cases. The average length of time from index surgery to revision was 16 years (range, 14-22 years). Ten patients underwent concomitant femoral component revision.

\section{Cage Design}

We constructed the 3-D prototypes in collaboration with the design and manufacturing industry using computeraided design software and rapid-prototyping techniques. Briefly, CT scans of the whole pelvis, taken at 1-mm intervals, were converted to 3-D digital models using specialized software (MIMICS ${ }^{\circledR}$ [Materialise Interactive Medical Image Control System]; Materialise, Leuven, Belgium). These digital models then were used to manufacture life-sized physical models using a laminated object manufacturing system called Dimension Elite (Stratasys Inc, Eden Prairie, MN, USA); the whole process could be completed in no more than 2 days. These rapid-prototype models then were used as the basis for varying the design according to the nature of the bone defect to achieve better initial stability. For instance, it might be decided to add two crests or a 3-D printed augment to the superior surface of the cage for stable support, expand the artificial iliac wing for rotational stability, or lengthen or reposition the artificial iliac braid to ensure enough screws could be used for firm fixation (Table 1).

The rotational center of the defective acetabulum had to be confirmed before cage design. This required drawing a single plane containing three points: the mid-point of the line connecting the anterosuperior iliac spines, that of the pubic symphysis, and that of the fifth lumbar vertebra. Then, a line perpendicular to the plane was drawn starting from the geometric center of the contralateral femoral head and extended isometrically, allowing for identification of the center of the diseased femoral head, which theoretically should correspond to the center of the ipsilateral femoral head (Fig. 1).

Once the center of the acetabulum was determined, the cage was designed according to the type of acetabular bone defect. The cages were made of grit-blasted titanium to facilitate ongrowth of the bone. In cases in which the supraacetabular bone defect was severe, the site and size of the crests or augment on the superior surface of the cage were tailored to the morphologic features of the individual bone defect. The crests were designed to ensure close contact with the remainder of the superior part of the ilium, thus ensuring vertical support and increasing rotational stability; the space between the crests allowed room for morselized bone allograft. The 3-D printed augments were made from titanium alloy using direct metal laser sintering. The top of each augment, which would be in contact with host bone, contained pores approximately 400 to $500 \mu \mathrm{m}$ in diameter to permit bone ingrowth, whereas the base was solid so it could be screwed to the cage (Fig. 2).

The appropriate position and number of screw holes in the artificial iliac wing or braid were determined using data on pelvic anatomy, such as the thickness of the ilium obtained from the CT scan and the rapid-prototyping model. The braid would be lengthened or repositioned as necessary to allow sufficient screws to be inserted for fixation. In cases in which the anterior or posterior column bone defect was especially severe, a wing would be substituted for the braid to allow better iliac fixation and rotational stability (Fig. 3); generally, an artificial wing would contain two rows of four to six holes for $6.5-\mathrm{mm}$ screws.

Other details of cage design could be customized as required. For example, an obturator hook under the inferior surface of the cage was preferred to an ischial flange because such a hook allows easier insertion at the time of surgery, permitting the surgeon to control the level of cage placement and bearing part of the load. The more open frame-style cage design was used in preference to the dome style seen in most custom triflanged cages because it allows convenient placement of a morselized bone graft behind the cage or between the crests.

Construction time for each cage was approximately 4 to 6 days, and the design of each completed cage was checked against the rapid-prototyping model before surgery.

\section{Surgical Procedures}

The revision procedures were performed mainly using the posterolateral approach (ZZ and KD). In certain cases in which the wing was too large to be inserted easily through the posterolateral approach, the Smith-Petersen approach would be used. We previously described our procedures in detail [18]. Briefly, after removal of the failed implant, all interface membranes in the acetabulum and areas of bone defect were removed, exposing the underlying acetabular morphologic features as modeled during rapid prototyping. The inferior margin of the acetabulum was exposed, allowing for placement of the fixation hook of the cage. The soft tissue was separated along the iliac wing with a periosteal elevator to allow proper positioning of the cage's iliac wing or braid. Care was taken to protect the superior 


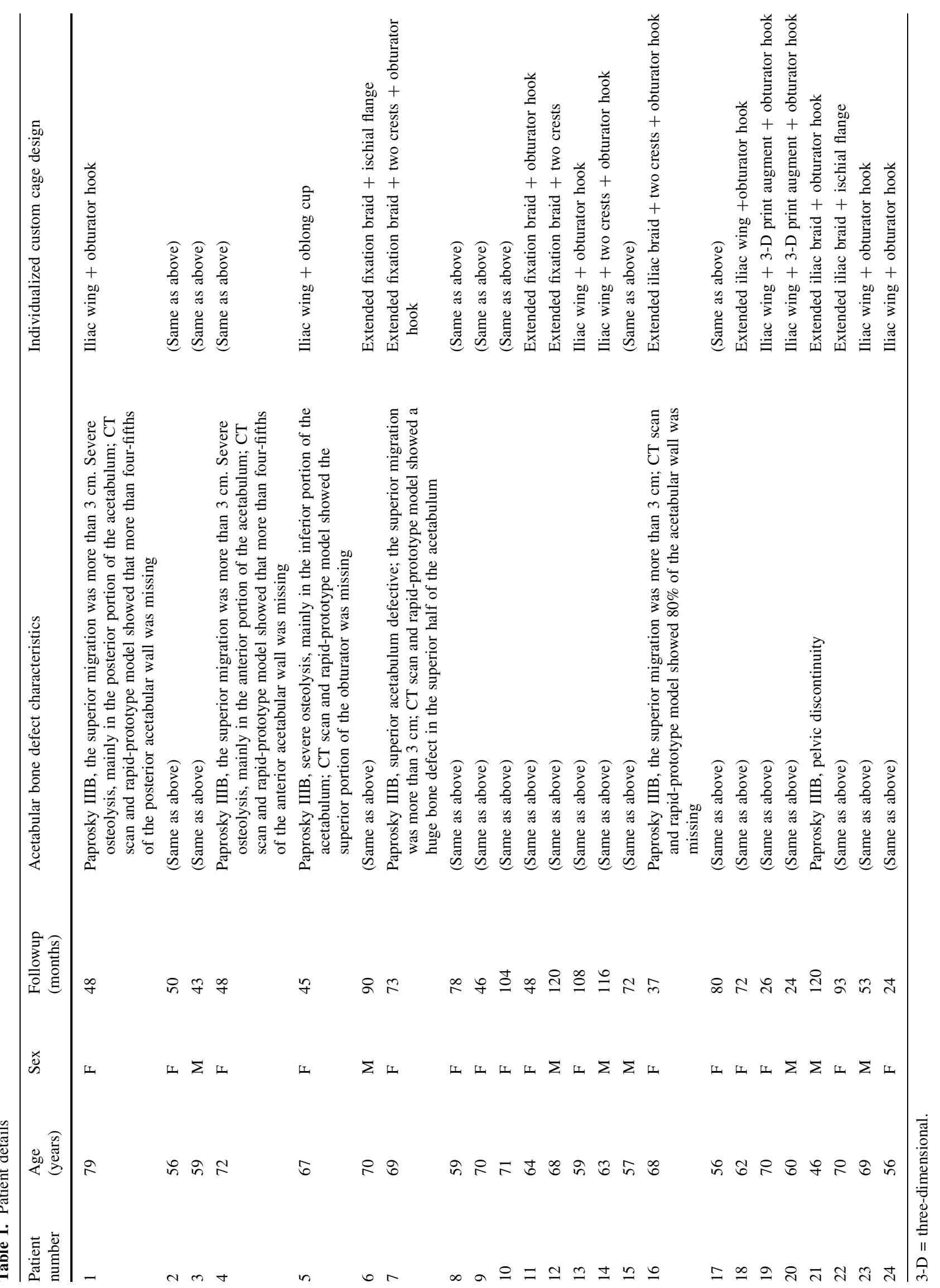




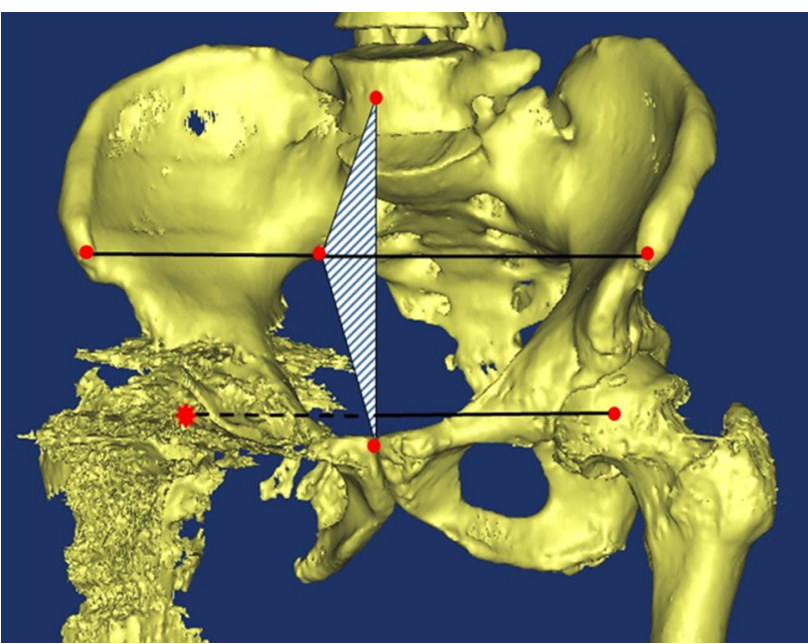

Fig. 1 The method for determining the rotational center of a severely defective acetabulum is shown. A single plane is drawn containing three points: the mid-point of the line connecting the anterosuperior iliac spines, the center of the pubic symphysis, and the mid-point of the fifth lumbar vertebra. Then, a line perpendicular to the plane is drawn starting from the geometric center of the healthy femoral head and extended isometrically, allowing identification of the center of the diseased femoral head.

gluteal artery and nerve during elevation of the gluteus minimus and medius from the iliac wing. The cage, with crests or augment as necessary, was positioned in the predefined site according to the rapid-prototyping model; then, the angles of anteversion and abduction were adjusted, and screws were inserted at the predefined site in a predefined number at the predefined angle. In all cases, allogeneic morselized bone grafts were used to completely fill the gap between the cage and host bone, either before or after cage placement. On completion of cage placement and bone grafts, a polyethylene liner was fixed at the normal angles of anteversion and abduction using bone cement.

\section{Postoperative Recovery and Assessment of Outcomes}

For the first 2 to 6 weeks after surgery, patients were limited to nonweightbearing or toe-touch weightbearing ambulation. They then graduated to partial weightbearing with crutches, and full weightbearing was allowed after 3 months.

All patients were examined clinically and radiographically at 6 and 12 weeks after surgery and once yearly thereafter. The Harris hip score was recorded at each followup. The Harris hip score at the latest followup was used for comparison with the score before surgery.

Measurements were made of the inclination of the cage and the hip rotation center (including the horizontal distance between the center of each hip and pubic symphysis and the vertical distance between the center of each hip and the line connecting the superior portions of the bilateral obturator foramina). The absolute difference of horizontal and vertical distances of the bilateral hip center was measured to assess the accuracy of the acetabulum center reconstruction. Radiograph magnification was calibrated using the known diameter of the artificial femoral head. One of the authors (HL) initially completed all measurements. Ten samples then were randomly selected and measured by another author (XQ), who was not involved in the patients' care, to test reliability. Agreement was good between the two authors (Cohen's unweighted $\kappa=0.93$ ).

Migration was assessed by comparing the patients' most recent radiographs with the immediate postoperative ones and looking for any movement of the cage or screws. Definite loosening was defined as acetabular migration of $2 \mathrm{~mm}$ or more in the horizontal or vertical direction, with implant rotation or screw breakage. Probable loosening was defined by a radiolucent line greater than $1 \mathrm{~mm}$ through all three zones of the acetabulum without migration, rotation, or screw breakage [19]. The grafted bone was considered to be integrated if trabeculae were continuous between the host bone and the grafts, and grafts were considered fully absorbed when the grafted bone visible immediately after surgery no longer was visible [17].

Surgical complications, revision procedures, and reoperations not treated by revision were collected by chart review.

\section{Statistical Analysis}

Statistical analysis was conducted with SPSS for Windows Version 11.5 (SPSS Inc, Chicago, IL, USA). Student's t-test for paired samples was used to compare the preoperative and postoperative Harris hip scores and the radiographic parameters between the revised and contralateral sides.

\section{Results}

Harris hip scores improved from a mean of 36 before surgery (SD, 8; range, 20-49) to 82 at latest followup (SD, 18 ; range, $60-96 ; \mathrm{p}<0.001)$. At this point, 18 of 24 patients $(75 \%)$ could walk unaided, five $(21 \%)$ used a cane, and one (4\%) used a walker.

Individualized custom cages resulted in generally reliable restoration of the hip center. The mean vertical distance was $25 \mathrm{~mm}$ (SD, $5 \mathrm{~mm}$; range, $19-40 \mathrm{~mm}$ ) on the revised side and $24 \mathrm{~mm}(\mathrm{SD}, 5 \mathrm{~mm}$; range, $18-40 \mathrm{~mm})$ on the contralateral side $(\mathrm{p}=0.265)$; the difference between bilateral sides was $0.4 \mathrm{~mm}$ ( $\mathrm{SD}, 3 \mathrm{~mm}$; range, -4.5 to $5 \mathrm{~mm}$ ). The mean horizontal distance was $106 \mathrm{~mm}$ (SD, 

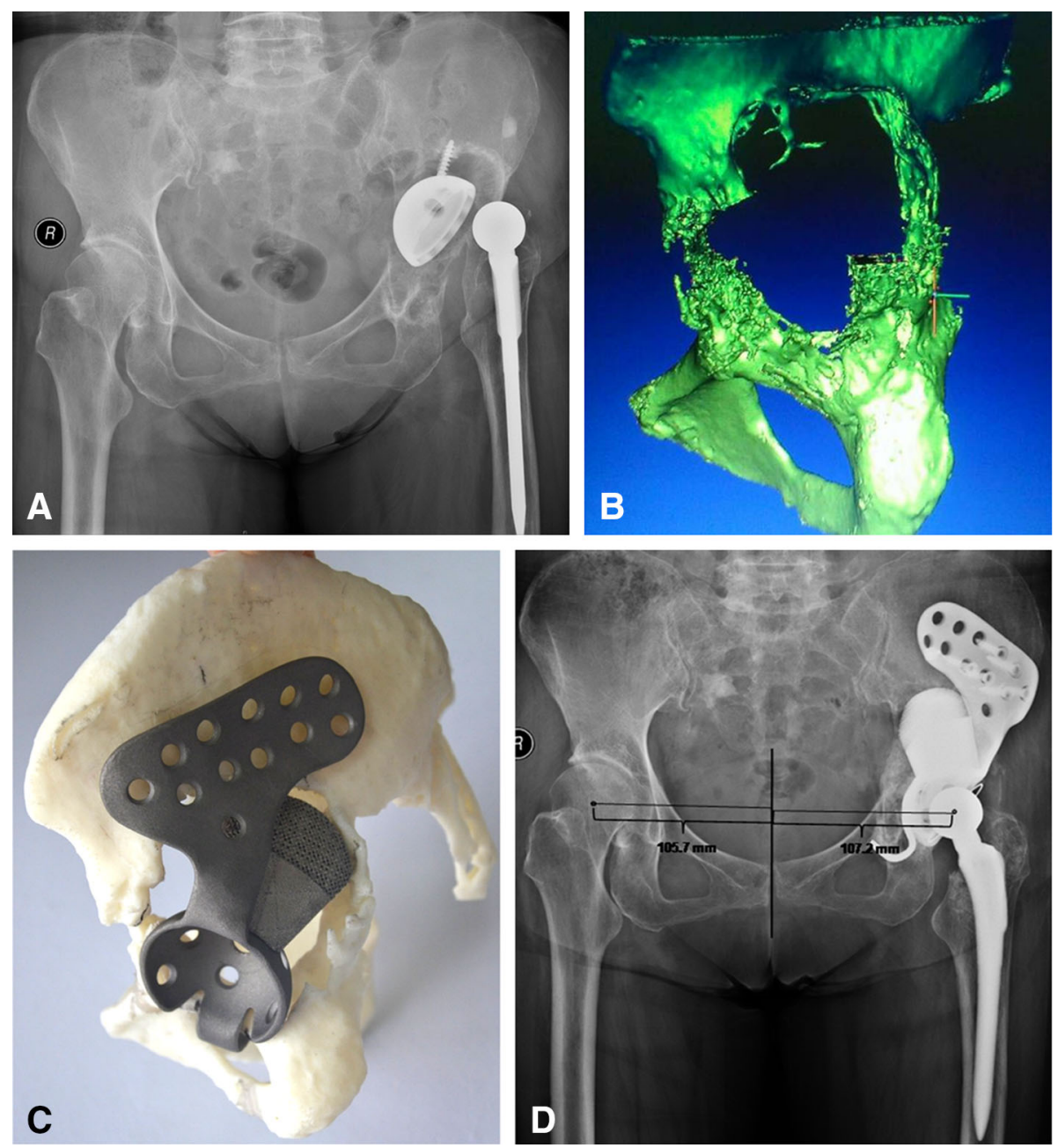

Fig. 2A-D (A) An AP radiograph shows prosthetic cup loosening with severe acetabular osteolysis 14 years postoperatively in a 70year-old woman. (B) A CT-based three-dimensional (3-D) reconstruction of the patient's acetabulum is shown. (C) An individualized custom cage with an artificial iliac wing, 3-D printed support

$9 \mathrm{~mm}$; range, $90-119 \mathrm{~mm}$ ) on the revised side and $109 \mathrm{~mm}$ (SD, $9 \mathrm{~mm}$; range, $94-123 \mathrm{~mm})$ on the other side $(\mathrm{p}=$ $0.75)$, and the difference was $-3 \mathrm{~mm}(\mathrm{SD}, 7 \mathrm{~mm}$; range, -19 to $8 \mathrm{~mm}$ ). Radiographic analysis showed the mean inclination of the cage was $46^{\circ}\left(\mathrm{SD}, 6^{\circ}\right.$; range, $\left.38^{\circ}-58^{\circ}\right)$.

No definite migration of any of the acetabular cups was observed. In one hip, however, a completely circumferential 2-mm radiolucent line was observed around the acetabulum without observable migration, rotation, or screw breakage, suggesting possible loosening of the cage. A radiolucent line was observed in one other hip, but it was only $1 \mathrm{~mm}$ wide, incomplete, and nonprogressive. The cancellous allograft appeared to become well incorporated in 23 of the 24 patients in whom the cage was judged to be radiographically stable (Fig. 4). augment, and obturator hook was constructed, and installation was simulated using the rapid-prototyping model. (D) A postoperative AP radiograph shows excellent restoration of the rotational center of the hip.

For the patient who needed a walker, the Trendelenburg test was positive, indicating possible injury to the superior gluteal nerve during exposure of the ilium. Infections developed in two hips. Diagnosis of both infections was made within several weeks of surgery (one deep infection and one superficial wound infection). The infections were treated with irrigation, débridement, vacuum-sealing drainage, and antibiotics. In both hips, the customized cage was retained and the infection resolved, with no recurrence at latest followup. One patient experienced a hip dislocation 4 days postoperatively when the hip flexed at an angle exceeding $90^{\circ}$; the dislocation resolved after closed reduction and 3 weeks of skin traction. At the end of the initial followup, the patient's Harris hip score was 86. No rerevisions were done. 

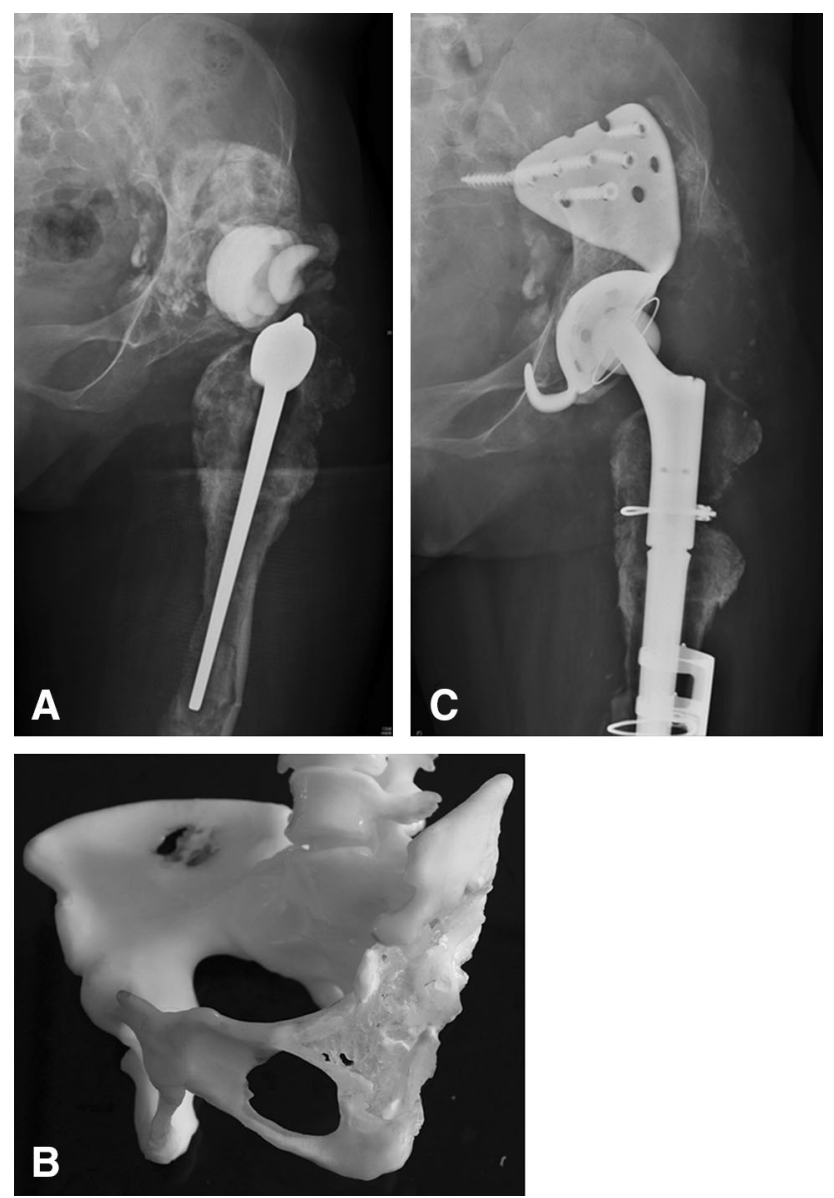

Fig. 3A-C (A) A radiograph shows prosthetic cup loosening with severe acetabular osteolysis 34 years postoperatively in a 56-year-old woman. (B) The rapid-prototyping model shows loss of the anterior and posterior columns and superior region of the acetabulum. (C) An individualized custom cage with an expanded iliac wing and obturator hook was used to achieve rotational stability.
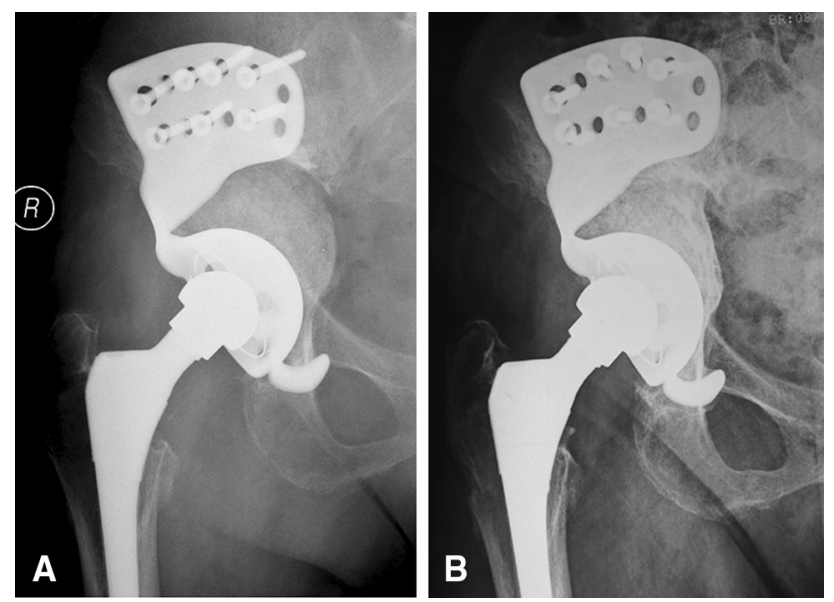

Fig. 4A-B (A) A radiograph obtained 6 weeks after surgery shows individualized custom cage reconstruction with morselized allograft placed medially. (B) Five years postoperatively, complete allograft incorporation was seen.

\section{Discussion}

Reconstruction of the acetabulum with a large defect poses a challenge at revision surgery. A key to success in these difficult cases is to obtain fixation on remaining autologous bone. The custom acetabular component has the reported advantage of being able to rigidly span large combined acetabular defects while having a custom fit to host bone, but the reports of this technique are few and the series are small $[7,20]$. We hoped to further clarify the rationale of the design and the advantages of using custom cages for complex acetabular revisions. We determined that individualized custom cages result in (1) improved Harris hip scores, (2) restoration of the hip center, and (3) a low frequency of surgical complications.

This study has several limitations. First, the sample size was small, and only 24 patients with Paprosky IIIB acetabular defects were included in our final sample. A study in a larger group of patients is necessary. Second, because of the complexity of the structure, the radiographic assessment of custom cages is more challenging than that of a hemispheric shell. As a result, we may have missed some custom cages that were loose or becoming loose. In addition, the study was retrospective and we were not able to compare this technique with other modern techniques such as cup-cage or triflange constructs while controlling for similar defects. Such a study would likely require a multiinstitutional approach. Previous studies have shown the primary disadvantages of custom cages to be their high cost in time and money [5, 10]. Although this approach was relatively economic at our center (CT scanning and model creation cost approximately USD 500, and the cost of custom cage manufacture, including the cup, screws, and polyethylene liner, was approximately USD 5000), this can vary widely from center to center, and our study was not a cost analysis. Future studies should compare the costs associated with various approaches with costs of complex acetabular revision, and the studies should consider reoperations and durability in their analyses.

The mean Harris hip score for our 24 patients increased from 36 preoperatively to 82 at an average followup 6 years later. Similarly, Holt and Dennis [15], who used custom triflange cups, reported Harris hip scores that increased from 39 preoperatively to 78 at an average last followup 4.5 years later. Taunton et al. [32] reported a Harris hip score of 75 at an average followup 5 years after surgery, and Wind et al. [36] reported an increase from 38 preoperatively to 63 at an average followup 3 years after surgery (Table 2) $[5,8,10,15,16,32,36]$. Our approach using individualized custom cages in patients with significant acetabular defects showed equal or better hip scores than the aforementioned series, for patients with comparable (or worse) defects and with longer followup. 


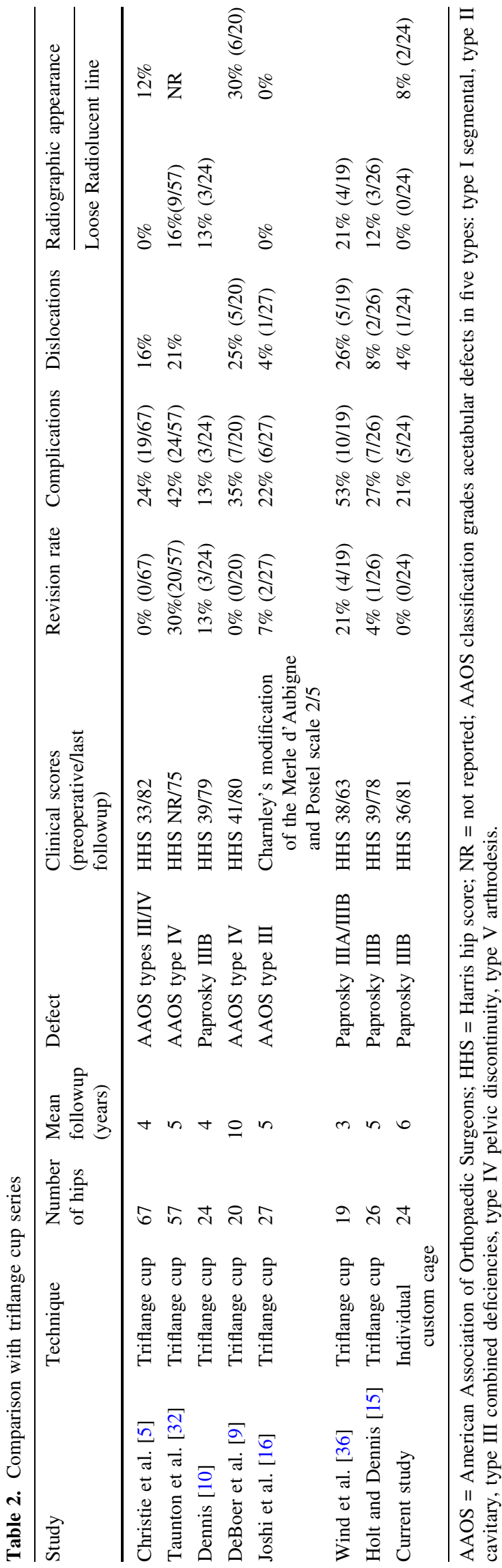

The individualized custom cages in our series differ from triflange cups in that the cages we used can be positioned more easily to restore the center of hip rotation and the inclination and anteversion of the cup. Once the appropriate position of the cage has been determined, our rapid-prototyping models indicate if the superior acetabulum provides sufficient contact and support for the cage; if not, a modified crest or 3-D printed augment can be added to the superior surface of the cage. Dislocation is a frequent problem with triflange cups, occurring in $16 \%$ to $26 \%$ of hips $[5,9,32,36]$. Dislocations are more common after difficult revision procedures like these because of component malposition, an absent or damaged abductor mechanism, or inadequate soft tissue tension [5, 15, 25]. In our study, only one of 24 patients experienced dislocation, which appears to be less than reported with other techniques $[5,9,15,32,36]$. We believe that our method's reasonably accurate restoration of the hip center and its control of the inclination and anteversion of the cage contributed to restoring the tension of the soft tissue and reducing impingement between the cage and femoral neck. Simulated in vitro insertion of the cage can help the clinician prepare for correct placement, which may aid him or her substantially during cage placement in vivo, reducing the incidence of dislocation.

A clear advantage of individualized custom cages is that surgeons can design them as required to obtain structural support from, and secure fixation to, remaining host bone. We observed only one case of suspicious loosening compared with loosening observed in as many as $21 \%$ of custom triflange cup procedures (customizable titanium acetabular components with ilial, ischial, and pubic flanges) in similar patients [36]. We also observed no definite migration and no components were revised. Some authors, however, have expressed concerns regarding the mechanical failure rate with complex acetabular revisions. Sporer et al. [31] reported a $37.5 \%$ failure rate after 2 to 8 years of followup for patients with massive acetabular defects treated with reconstruction cages. Loss of effective host bone contact and support for the cage is the leading cause of mechanical failure [3, 5, 6, 15, 26, 31]. Although the use of structural bone grafts that provide auxiliary support initially might achieve cage stability, subsequent bone resorption may cause loosening and displacement of the cage. Udomkiat et al. [33] used metal rings to perform acetabular revision in 64 patients and conducted a 5-year followup study that showed patient susceptibility to implant loosening and displacement is increased substantially if more than $60 \%$ of the weightbearing area is filled with bone graft. Paprosky et al. [22], studying a sample of 11 patients who underwent cage revision for complex bone defects, reported that all seven failures in their cohort were caused by ineffective bone support. Our use of the frame- 
style and crest design also allows for more convenient introduction of morselized allograft materials. According to previous studies [5, 24], morselized allograft exhibits excellent osteointegration along with good medium- to long-term results provided implant stability on host bone can be achieved. In the current study, cancellous allograft was judged incorporated in the 23 of 24 patients in whom the cage was thought to be stable, indicating a good longterm result.

We had one case of suspected superior gluteal nerve injury in this series. Substantial exposure of the ilium is required for accurate placement of the custom cage. This risks injury to the superior gluteal nerve and subsequently increases the risk of hip dislocation. Several methods can be used to reduce the risks. First, a greater trochanteric osteotomy or extended trochanteric osteotomy may be used to relieve tension on the superior gluteal neurovascular pedicle during insertion of the cage or stem removal. Second, the screws in the anterior parts of the wing can be inserted percutaneously. Third, the Smith-Petersen approach can be used in certain cases in which the wing is too big to be inserted easily through the posterolateral approach; however, the dissection of the soft tissue will be more extensive.

We believe individualized custom cages provide a viable solution for difficult acetabular reconstructions involving severe bone loss by permitting stable implant fixation on host bone, allowing maintenance of a closer-tonormal hip center, and restoring periprosthetic bone stock through the frame-style cage with morselized cancellous allograft. Short-term results show excellent mechanical stability and improved hip scores in this small, singlecenter series, but the long-term outcomes associated with this technique in comparison to other options remain to be determined.

\section{References}

1. Abolghasemian M, Tangsaraporn S, Drexler M, Barbuto R, Backstein D, Safir O, Kuzyk P, Gross A. The challenge of pelvic discontinuity: cup-cage reconstruction does better than conventional cages in mid-term. Bone Joint J. 2014;96:195-200.

2. Amenabar T, Rahman WA, Hetaimish BM, Kuzyk PR, Safir OA, Gross AE. Promising mid-term results with a cup-cage construct for large acetabular defects and pelvic discontinuity. Clin Orthop Relat Res. 2015 Feb 25. [Epub ahead of print]

3. Berry DJ, Müller ME. Revision arthroplasty using an anti-protrusio cage for massive acetabular bone deficiency. J Bone Joint Surg Br. 1992;74:711-715.

4. Chiang PP, Burke DW, Freiberg AA, Rubash HE. Osteolysis of the pelvis: evaluation and treatment. Clin Orthop Relat Res. 2003;417:164-174.

5. Christie MJ, Barrington SA, Brinson MF, Ruhling ME, DeBoer DK. Bridging massive acetabular defects with the triflange cup: 2- to 9-year results. Clin Orthop Relat Res. 2001;393:216-227.
6. D'Antonio JA, Capello WN, Borden LS, Bargar WL, Bierbaum BF, Boettcher WG, Steinberg ME, Stulberg SD, Wedge JH. Classification and management of acetabular abnormalities in total hip arthroplasty. Clin Orthop Relat Res. 1989;243:126-137.

7. Dai KR, Zhu ZA, Sun YH, Shang XF, Zhang J. The reconstruction of massive bone defect in acetabulum in revision surgery. Chin J Orthop. 2001;21:332-336.

8. Dearborn JT, Harris WH. Acetabular revision arthroplasty using so-called jumbo cementless components: an average 7-year follow-up study. J Arthroplasty. 2000;15:8-15.

9. DeBoer DK, Christie MJ, Brinson MF, Morrison JC. Revision total hip arthroplasty for pelvic discontinuity. J Bone Joint Surg Am. 2007;89:835-840.

10. Dennis DA. Management of massive acetabular defects in revision total hip arthroplasty. J Arthroplasty. 2003;18(3 suppl 1):121-125.

11. Gill TJ, Sledge JB, Müller ME. The management of severe bone loss using structural allograft and acetabular reinforcement devices. J Arthroplasty. 2000;15:1-7.

12. Grappiolo G, Loppini M, Longo UG, Traverso F, Mazziotta G, Denaro V. Trabecular metal augments for the management of Paprosky type III defects without pelvic discontinuity. $J$ Arthroplasty. 2015;30:1024-1029.

13. Haddad FS, Shergill N, Muirhead-Allwood SK. Acetabular reconstruction with morsellized allograft and ring support: a medium-term review. J Arthroplasty. 1999;14:788-795.

14. Harris WH. Wear and periprosthetic osteolysis: the problem. Clin Orthop Relat Res. 2001;393:66-70.

15. Holt GE, Dennis DA. Use of custom triflanged acetabular components in revision total hip arthroplasty. Clin Orthop Relat Res. 2004;429:209-214.

16. Joshi AB, Lee J, Christensen C. Results for a custom acetabular component for acetabular deficiency. J Arthroplasty. 2002; 17:643-648.

17. Knight JL, Fujii K, Atwater R, Grothaus L. Bone-grafting for acetabular deficiency during primary and revision total hip arthroplasty: a radiographic and clinical analysis. J Arthroplasty. 1993;8:371-382.

18. Li H, Wang L, Mao Y, Wang Y, Dai K, Zhu Z. Revision of complex acetabular defects using cages with the aid of rapid prototyping. J Arthroplasty. 2013;28:1770-1775.

19. Massin P, Schmidt L, Engh CA. Evaluation of cementless acetabular component migration: an experimental study. $J$ Arthroplasty. 1989;4:245-251.

20. Munjal S, Leopold SS, Kornreich D, Shott S, Finn HA. CTgenerated 3-dimensional models for complex acetabular reconstruction. J Arthroplasty. 2000;15:644-653.

21. Paprosky WG, Perona PG, Lawrence JM. Acetabular defect classification and surgical reconstruction in revision arthroplasty: a 6-year follow-up evaluation. J Arthroplasty. 1994;9:33-44.

22. Paprosky WG, Sporer SS, Murphy BP. Addressing severe bone deficiency: what a cage will not do. J Arthroplasty. 2007;22(4 suppl 1):111-115.

23. Perka C, Ludwig R. Reconstruction of segmental defects during revision procedures of the acetabulum with the Burch-Schneider anti-protrusio cage. J Arthroplasty. 2001;16:568-574.

24. Peters CL, Curtain M, Samuelson KM. Acetabular revision with the Burch-Schneider antiprotrusio cage and cancellous allograft bone. J Arthroplasty. 1995;10:307-312.

25. Peters CL, Miller M, Erickson J, Hall P, Samuelson K. Acetabular revision with a modular antiprotrusio acetabular component. J Arthroplasty. 2004;19(7 suppl 2):67-72.

26. Pieringer H, Auersperg V, Böhler N. Reconstruction of severe acetabular bone-deficiency: the Burch-Schneider antiprotrusio cage in primary and revision total hip arthroplasty. J Arthroplasty. 2006;21:489-496. 
27. Pope D, Blankenship S, Jones G, Robinson BS, Maloney WJ, Paprosky WG, Ries MD, Saleh KJ. Maximizing function and outcomes in acetabular reconstruction: segmental bony defects and pelvic discontinuity. Instr Course Lect. 2014;63:187-197.

28. Saleh KJ, Jaroszynski G, Woodgate I, Saleh L, Gross AE. Revision total hip arthroplasty with the use of structural acetabular allograft and reconstruction ring: a case series with a 10-year average follow-up. J Arthroplasty. 2000;15:951-958.

29. Schatzker J, Wong MK. Acetabular revision: the role of rings and cages. Clin Orthop Relat Res. 1999;369:187-197.

30. Schreurs BW, Thien TM, de Waal Malefijt MC, Buma P, Veth RP, Slooff TJ. Acetabular revision with impacted morselized cancellous bone graft and a cemented cup in patients with rheumatoid arthritis: three to fourteen-year follow-up. $J$ Bone Joint Surg Am. 2003;85:647-652.

31. Sporer SM, Paprosky WG, O'Rourke M. Managing bone loss in acetabular revision. J Bone Joint Surg Am. 2005;87:1620-1630.

32. Taunton MJ, Fehring TK, Edwards P, Bernasek T, Holt GE, Christie MJ. Pelvic discontinuity treated with custom triflange component: a reliable option. Clin Orthop Relat Res. 2012;470:428-434.

33. Udomkiat P, Dorr LD, Won YY, Longjohn D, Wan Z. Technical factors for success with metal ring acetabular reconstruction. J Arthroplasty. 2001;16:961-969.

34. van Egmond N, De Kam DC, Gardeniers JW, Schreurs BW. Revisions of extensive acetabular defects with impaction grafting and a cement cup. Clin Orthop Relat Res. 2011;469:562-573.

35. van Haaren AH, Heyligers IC, Alexander FG, Wuisman PI. High rate of failure of impaction grafting in large acetabular defects. J Bone Joint Surg Br. 2007;89:296-300.

36. Wind MA Jr, Swank ML, Sorger JI. Short-term results of a custom triflange acetabular component for massive acetabular bone loss in revision THA. Orthopedics. 2013;36:e260-e265.

37. Winter E, Piert M, Volkmann R, Maurer F, Eingartner C, Weise $\mathrm{K}$, Weller S. Allogeneic cancellous bone graft and a BurchSchneider ring for acetabular reconstruction in revision hip arthroplasty. J Bone Joint Surg Am. 2001;83:862-867. 